\title{
Pulse Measurements of the Frequency Response of a Photoacoustic Cell
}

\author{
M. Suchenek • T. Starecki
}

Received: 11 April 2010 / Accepted: 1 March 2011 / Published online: 17 March 2011

(C) The Author(s) 2011. This article is published with open access at Springerlink.com

\begin{abstract}
The determination of the frequency response of a photoacoustic cell is a typical activity during design and adjustment of a photoacoustic setup. The most common method of such measurements is based on point-by-point checking of the amplitude of the photoacoustic signal at different modulation frequencies. Such an approach can be time-consuming, especially if a lot of measurements at low modulation frequencies are to be performed. The article presents a method based on investigation of a photoacoustic response to short square pulse stimulation instead of continuous modulation of the light source. The method allows for measurements of the frequency response to be substantially shortened. A theoretical description and properties of the pulse measurement method are discussed and illustrated with a comparison of experimental results obtained from both frequency response measurement methods.
\end{abstract}

Keywords Frequency response $\cdot$ Photoacoustics $\cdot$ Pulse measurements

\section{Introduction}

Photoacoustic investigations require quite often measurements at relatively low signal levels, which sometimes are buried deeply in noise. One of the techniques commonly applied to increase the level of the photoacoustic signal is its amplification by means of a resonance cell. Even if resonance of the cell is not used for the purpose of signal amplification, the frequency response of the cell should also be known to evaluate the suitability of the cell for particular measurements and to take it into account in data processing in a target application. This means that frequency response measurements

M. Suchenek $(\varangle) \cdot$ T. Starecki

Institute of Electronic Systems, Warsaw University of Technology, Nowowiejska 15/19,

00-665 Warsaw, Poland

e-mail: M.Suchenek@ise.pw.edu.pl 
of photoacoustic cells are performed mainly in two cases. The first case is photoacoustic cell research — during prototyping, and investigating acoustic properties of many different cells. In such research quite often tens or even hundreds of different cell configurations have to be investigated $[1,2]$, and their frequency responses have to be measured in a wide frequency range. This step cannot be skipped and based on pure modeling, as even in the case of relatively simple cells, their frequency responses can be complex [3-5] and quite often different from the theoretical predictions $[1,2]$. The second case is resonance tracking/locking during target measurements, especially if the cell has a high value of the Q-factor. In such a case even small detuning from the resonance frequency results is a significant drop of the signal amplitude [6]. In that kind of application of the frequency response measurements, it is usually enough to scan a much smaller frequency range. In both mentioned cases fast frequency response measurements are advantageous. The presented pulse measurement method was primarily aimed at the applications of the first kind, but it can be also used as an alternative to many existing methods of resonance locking/tracking, which are based on chirp excitation [7], locking to another resonance frequency [8], self-oscillation [9], etc.

Usually the frequency response is obtained point-by-point, by excitation of the resonator at a single frequency and measuring the amplitude of its output signal [10-12]. However, if we assume that the measured acoustic resonator is a linear and stationary circuit, the frequency response can be determined in another way, which is equivalent from the point of view of signal analysis. The resonator can be excited with a signal composed of all the frequencies to be measured in the frequency response, because linearity of the circuit means that the result of excitation with a complex signal is equivalent to the superposition of responses to separated signal components. Such a method is quite commonly used in the field of electronics, e.g., in determination of the bandwidth of oscilloscopes by means of the analysis of its step or pulse response $[13,14]$. This article discusses some aspects of such an approach applied to measurements of the frequency responses of photoacoustic cells.

\section{Theoretical Background}

According to the second mentioned method, it is possible to determine the frequency response of a photoacoustic cell in a single measurement. The required and sufficient condition is the use of an excitation signal that would contain frequencies covering the range of interest with reasonable resolution. The simplest signal (in terms of its generation) that would fulfill such a requirement is a short square pulse. Although pulse operation has been used in photoacoustic measurements for many years $[15,16]$ and there are many papers describing theoretical and practical aspects of pulsed photoacoustic operation $[5,6,15,17-20]$, the use of a pulse excitation for the purpose of frequency response measurements requires some additional considerations.

Harmonics of a square pulse exist at all multiples of its fundamental frequency, and their amplitudes are given by [21]

$$
X(\omega)=\frac{2 A}{\omega} \sin \left(\frac{\tau}{2} \omega\right)
$$




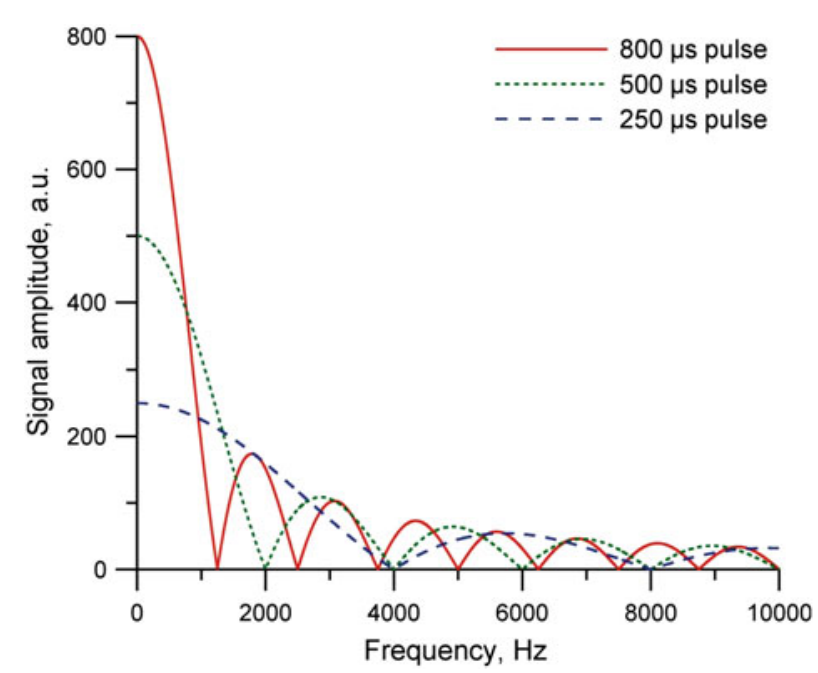

Fig. 1 Envelope of the pulse signal spectrum given by Eq. 1 for different values of the pulse length $\tau$ $(800 \mu \mathrm{s}, 500 \mu \mathrm{s}, 250 \mu \mathrm{s})$ and period $T=500 \mathrm{~ms}$

where $A$ is the amplitude of the pulse; $\tau$ is the pulse length; $\omega=2 \pi n / T$, where $n=1,2, \ldots$; and $T$ is the pulse repetition period.

Hence, the frequency resolution (number of harmonics within the same frequency range) in the case of square pulse excitation depends on the period of the pulse. Thus, the pulse repetition frequency should be chosen as

$$
f_{0} \leq \Delta f
$$

where $\Delta f$ is the required maximum frequency step in the frequency response to be measured.

A few examples of the pulse signal spectrum as given by Eq. 1 for different values of the pulse length $\tau$ and period $T=500 \mathrm{~ms}$ are given in Figs. 1 and 2 .

Figures 1 and 2 show clearly that for longer pulses, amplitudes of low frequency harmonics are greater, but they decrease faster with the frequency. Hence, the pulse length should be selected according to the frequency range of interest. On one hand, it should be long enough to obtain possibly high amplitudes at lower frequencies, but on the other hand, the pulse cannot be too long to keep the amplitude of harmonics at the end of the measure frequency range also at a reasonable level. Another option is the use of a few pulses of different lengths for the measurements, but such an approach would reduce one of the main advantages of the pulse method, i.e., the simplicity of the setup. It should be also mentioned that characteristics given in Figs. 1 and 2 were calculated with the assumption of the same amplitude of the pulse in all cases. But, in the case of some semiconductor light sources, the instantaneous optical output power can be increased if the pulse is shortened, thus leading to a substantial increase of all the harmonics [19]. 


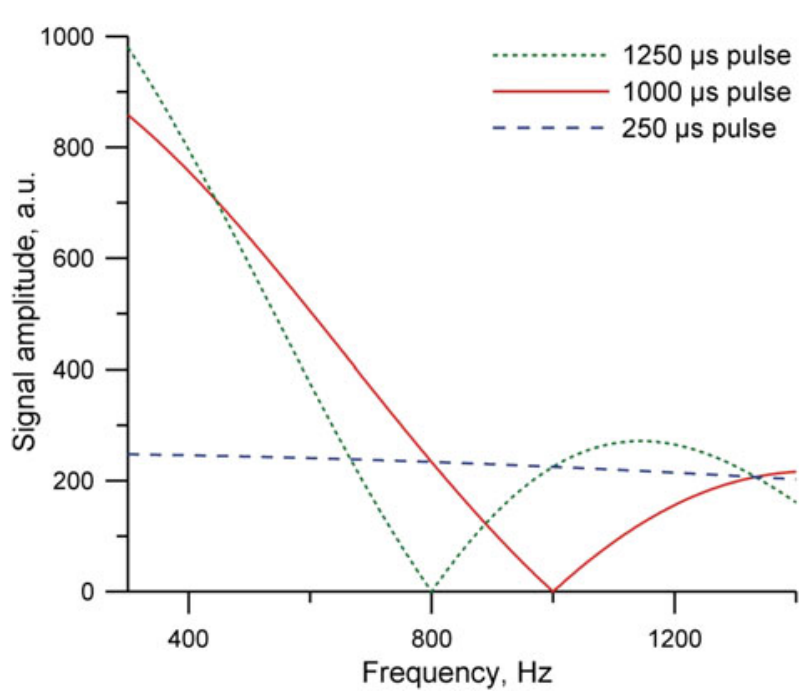

Fig. 2 Envelope of the pulse signal spectrum given by Eq. 1 for different values of the pulse length $\tau$ $(250 \mu \mathrm{s}, 1000 \mu \mathrm{s}, 1250 \mu \mathrm{s})$ and period $T=500 \mathrm{~ms}$ used in the measurements

\section{Measurements and Calculations}

In the discussed method, the whole frequency response can be calculated from a single measurement. In order to do this, the output signal from the excited resonator should be digitally recorded. Once such a vector of samples is available, it should be processed to get amplitudes of all the harmonics to be included in the frequency response. This can be done in a few ways: the amplitude at every single frequency can be calculated from the collected samples independently by means of discrete Fourier transform (DFT) or least-squares (LS) methods, which are equivalent in such a case [22], but fast Fourier transform (FFT) method will have better numerical efficiency in the discussed application. Once the signal is converted into the frequency domain, the calculated frequency response must be corrected by taking into consideration that obtained values of the harmonics reflect the relationship given in Eq. 1 and shown in Figs. 1 and 2. The necessity of such a correction of the measured frequency response is not a problem, because the algorithm is simple and the calculations can be executed very fast. The bigger difficulty is introduced by the substantial amount of data that must be recorded and analyzed, which results directly from the Nyquist-Shannon theorem, stating that the sampling frequency must be at least a factor of two greater than the highest frequency included in the frequency response. For example, a $2 \mathrm{kHz}$ frequency response requires a $4 \mathrm{kHz}$ sampling frequency which gives 2 samples per period at $2 \mathrm{kHz}$. Better accuracy gives sampling with more than 2 samples per period. In the experiment, we obtained 32000 samples with 3 bytes per sample, which required $96 \mathrm{kB}$ RAM. This is definitely not much from the point of view of the memory resources in modern computers or digital signal processors, but it can be a serious limit in the case of many microcontrollers. Preliminary measurements were performed with a low-power light source (single $\mathrm{mW}$ of optical power). This resulted in a relatively low photoacoustic 
signal, which required averaging. After replacing the preliminary light source with a $3 \mathrm{~W}$ LED diode, it was possible to perform the pulse frequency response measurements based on signal recording during a single light pulse period.

\section{Experimental Results}

The proposed method of the pulse measurement of the frequency response of a photoacoustic cell was experimentally verified. The measured cell was a photoacoustic Helmholtz resonator (Fig. 3). Sample and microphone cavities were $1 \mathrm{~cm}^{3}$ each, while the interconnecting duct was $25 \mathrm{~mm}$ in length and $2 \mathrm{~mm}$ in diameter. The absorbing substance was a thin layer of a carbon black powder placed on the bottom of the sample cavity.

At first, the frequency response of the cell was measured in the traditional way, i.e., point-by-point, with excitation of the resonator at frequencies starting at $200 \mathrm{~Hz}$ and ending at $2 \mathrm{kHz}$, with a step of approximately $2 \mathrm{~Hz}$. Then horizontal resolution in all these measurements was set to 16 samples per period of the recorded signal. The described measurements took $8 \mathrm{~min}, 45 \mathrm{~s}$ including signal acquisition time and data transmission time.

The second experiment was based on the short pulse excitation. In this case, stimulation of the cell was done with light pulses $250 \mu \mathrm{s}$ long, repeated every $500 \mathrm{~ms}$. As a result, the frequency step in this measurement was also $2 \mathrm{~Hz}$, but measurements took just approximately $6 \mathrm{~s}$ with most of the time spent on the data transmission, as the signal recording time was only $500 \mathrm{~ms}$, i.e., a single light pulse period (no signal averaging was used). In order to show what happens in the case of incorrect selection of the pulse length, similar measurements with pulses of $1000 \mu$ s and $1250 \mu$ s were performed.

A comparison of the frequency responses obtained with the step-by-step method and uncorrected pulse measurements is presented in Fig. 4. It can be easily noticed that only one of the responses measured with the pulse method is in good agreement

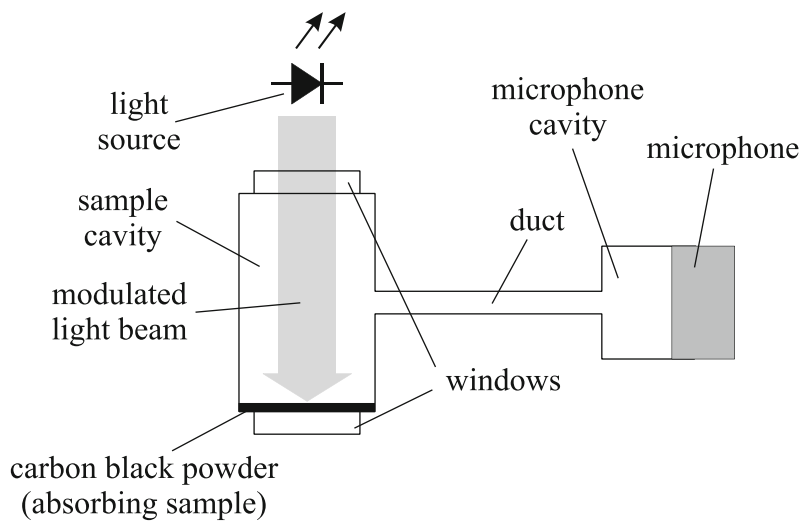

Fig. 3 Sketch of the photoacoustic cell used for experimental verification of the proposed pulse method of the frequency response measurements 


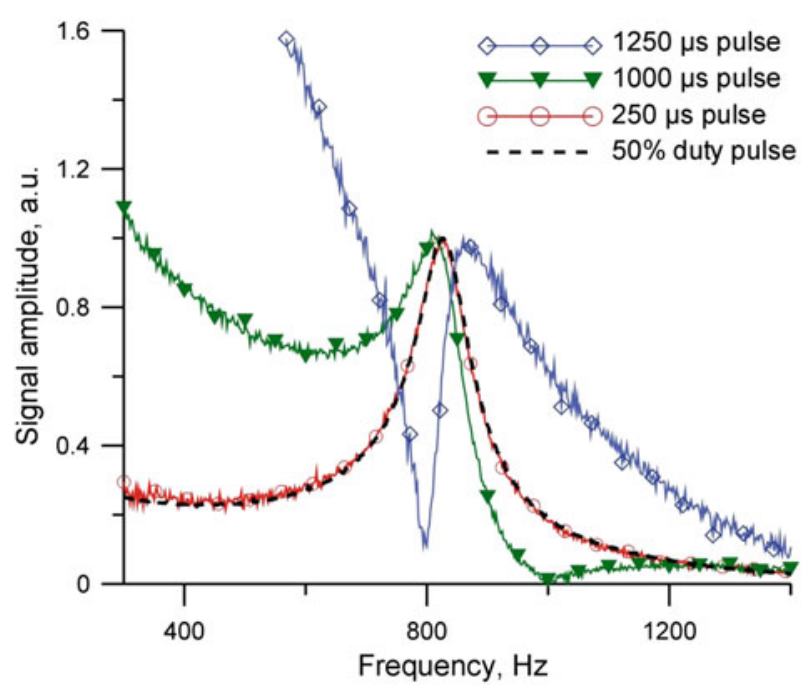

Fig. 4 Comparison of the frequency responses obtained experimentally with traditional (point-by-point) and pulse measurement methods (pulse measured responses uncorrected)

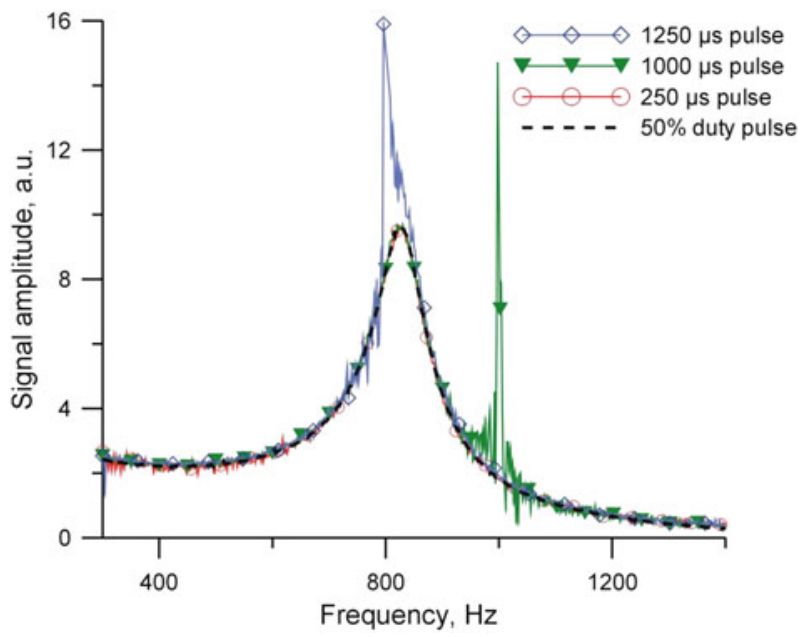

Fig. 5 Comparison of the frequency responses obtained experimentally with traditional (point-by-point) and pulse measurement methods (pulse measured responses corrected)

with the step-by-step measurements. This is due to the fact that only one of the stimulating pulses has a relatively uniform spectrum in the frequency range of interest (Fig. 2), and the frequency responses presented in Fig. 4 are uncorrected. Corrected frequency responses are given in Fig. 5. Although such corrected pulse-measured frequency responses show generally better agreement with the traditional measurements, it is clearly visible that some of them show artifacts around the frequencies which correspond to zeros of their spectra from Fig. 3. This proves that the stimulation pulse parameters (its length and repetition frequency) should be selected carefully. It can be 
also easily noticed that the frequency response measured with $250 \mu$ s pulse excitation presented in Fig. 5 is nearly identical with the point-by-point measured one. Slight differences result mainly from the amplifier's circuit nonlinear response, the nonideal shape of the square stimulation pulse, and errors introduced by digital recording $[23,24]$. But all the mentioned error sources can be considered as non-critical, as difference of the Q-factor values of the photoacoustic cell used in the experiments obtained with the two methods is at the level of approximately $1 \%$.

\section{Conclusions}

The proposed method of the pulse measurement of the photoacoustic cell frequency response has several advantages over traditional point-by-point measurements. One of them is a much simpler measurement setup, as the pulse method requires a generator producing square pulses of a fixed length and fixed repetition frequency instead of a frequency-adjustable one, which is needed in the traditional frequency response measurements. The pulse method is particularly suitable for use with semiconductor light sources, especially pulse lasers. Another important advantage of the described method is a much shorter time of the measurements - in the presented experiments, use of the pulse methods could be used to shorten the measurements to just a few seconds. The main drawback of the pulse method is the smaller amplitudes of the harmonic components in comparison to the traditional point-by-point measurement method. However, this is not a problem if the measurements are performed with moderately or strongly absorbing specimens. Hence, the pulse measurement of the frequency response of photoacoustic cells should be considered a valuable method in many practical applications.

Open Access This article is distributed under the terms of the Creative Commons Attribution Noncommercial License which permits any noncommercial use, distribution, and reproduction in any medium, provided the original author(s) and source are credited.

\section{References}

1. T. Starecki, J. Acoust. Soc. Am. 122, 2118 (2007)

2. M. Suchenek, Proc. SPIE Int. Soc. Opt. Eng. 6937, 693711 (2007)

3. A. Miklós, C. Brand, A. Winkler, P. Hess, J. Phys. IV C 7, 781 (1994)

4. M. Nägele, M.W. Sigrist, Appl. Phys. B 70, 895 (2000)

5. S. Schäfer, A. Miklós, P. Hess, Appl. Opt. 36, 3202 (1997)

6. A. Miklós, P. Hess, Z. Bozóki, Rev. Sci. Instrum. 72, 1937 (2001)

7. M. Szakáll, A. Varga, A. Pogány, Z. Bozóki, G. Szabó, Appl. Phys. B 94, 691 (2009)

8. G.Z. Angeli, Z. Bozóki, A. Miklós, A. Lörincz, A. Thöny, M.W. Sigrist, Rev. Sci. Instrum. 62, 810 (1991)

9. T. Borowski, T. Starecki, Eur. Phys. J. Spec. Top. 153, 439 (2008)

10. R. Kastle, M.W. Sigrist, Appl. Phys. B 63, 389 (1996)

11. O. Nordhaus, J. Pelzl, Appl. Phys. A 25, 221 (1981)

12. V. Zeninari, V.A. Kapitanov, D. Courtois, Yu.N. Ponomarev, Infrared Phys. Technol. 40, 1 (1999)

13. J.R. Andrews, Proc. IEEE 66, 414 (1978)

14. J.J. Blair, IEEE Trans. Instrum. Meas. 56, 807 (2007)

15. L.C. Aamodt, J.C. Murphy, J. Appl. Phys. 49, 3036 (1978)

16. M.F. Cox, G.N. Coleman, Anal. Chem. 53, 2034 (1981) 
17. A. Domanowska, R.J. Bukowski, Int. J. Thermophys. 30, 1536 (2009)

18. R.J. Bukowski, A. Domanowska, J. Phys. IV 109, 9 (2003)

19. T. Starecki, Acta. Phys. Pol. A 114, A-205 (2008)

20. R. Bartlome, M. Kaučikas, M.W. Sigrist, Appl. Phys. B 96, 561 (2009)

21. A. Papoulis, Signal Analysis (McGraw-Hill, New York, 1977), p. 62

22. T. Starecki, T. Owczarek, Proc. SPIE Int. Soc. Opt. Eng. 6937, 693710 (2007)

23. J.J. Blair, IEEE Trans. Instrum. Meas. 47, 345 (1997)

24. J.P. Deyst, T.M. Souders, IEEE Trans. Instrum. Meas. 45, 378 (1996) 